\title{
A hybrid Tabu search-simulated annealing method to solve quadratic assignment problem
}

\author{
Mohamad Amin Kaviani ${ }^{a^{*}}$, Mehdi Abbasi ${ }^{\mathrm{b}}$, Bentolhoda Rahpeyma ${ }^{\mathrm{b}}$ and Mohamad Mehdi Yusefi
}

\begin{tabular}{|c|c|}
\hline$\overline{C H R O N ~ I ~ C ~ L E ~}$ & A B S T R A C T \\
\hline $\begin{array}{l}\text { Article history: } \\
\text { Received October } 15,2013 \\
\text { Accepted January 24, } 2014 \\
\text { Available online } \\
\text { February } 232014 \\
\text { Keywords: } \\
\text { Hybrid optimization } \\
\text { Simulated annealing } \\
\text { Tabu search } \\
\text { Quadratic assignment problem } \\
\text { Meta heuristic methods }\end{array}$ & $\begin{array}{l}\text { Quadratic assignment problem (QAP) has been considered as one of the most complicated } \\
\text { problems. The problem is NP-Hard and the optimal solutions are not available for large-scale } \\
\text { problems. This paper presents a hybrid method using tabu search and simulated annealing } \\
\text { technique to solve QAP called TABUSA. Using some well-known problems from QAPLIB } \\
\text { generated by Burkard et al. (1997) [Burkard, R. E., Karisch, S. E., \& Rendl, F. (1997). } \\
\text { QAPLIB-a quadratic assignment problem library. Journal of Global Optimization, 10(4), 391- } \\
\text { 403.], two methods of TABUSA and TS are both coded on MATLAB and they are compared in } \\
\text { terms of relative percentage deviation (RPD) for all instances. The performance of the proposed } \\
\text { method is examined against Tabu search and the preliminary results indicate that the hybrid } \\
\text { method is capable of solving real-world problems, efficiently. }\end{array}$ \\
\hline
\end{tabular}

\section{Introduction}

Koopmans and Beckman (1957) are believed to be the first who introduced the quadratic assignment problem (QAP) in the context of locating "indivisible economic activities". The primary objective of QAP problem is to assign a set of facilities to a set of locations such that the total assignment cost is minimized. The assignment cost for a pair of facilities is considered as a function of the flow between the facilities and the distance between the locations of the facilities. Ahmed (2013) presented a new reformulation of the problem and developed a Lexisearch Algorithm (LSA) to obtain exact optimal solution to this problem. He performed a comparative study to show the efficiency of the algorithm against an existing algorithm for some medium sized instances from the QAP library, QAPLIB (Burkard et al., 1997; Burkard, 2013).

\footnotetext{
* Corresponding author.

E-mail addresses: aminkaviani1366@yahoo.com $\quad$ (M.A. Kaviani) 
Forghani and Mohammadi (2012) presented an integrated quadratic assignment and continuous facility layout problem. They obtained the arrangement of facilities within the departments through the QAP. They presented mathematical model as a mixed-integer programming (MIP) to minimize total material handling cost. In addition, they presented a heuristic method to solve the problem for large-scale problems and using several illustrative numerical examples, the performance of the model was examined. Tasgetiren et al. (2013) presented some metaheuristics to solve QAP problems. Tseng and Liang (2006) presented a hybrid metaheuristic for the quadratic assignment problem. Wang (2007) applied Tabu search to solve QAP problem.

\section{The proposed study}

\subsection{Problem statement}

In quadratic assignment problem, we are concerned with assignment of two facilities $i$ and $j$ in two possible places of $k$ and $l$. Let $x_{i k}$ be a binary variable, which is one if facility $i$ is located in place $k$ and zero, otherwise. In addition, Let $x_{j l}$ be a binary variable, which is one if facility $j$ is located in place $l$ and zero, otherwise. Let $c_{i j k l}$ be the cost of assigning location $i$ in place $k$ and location $j$ in place $l$. Therefore, the proposed study considers the following,

(QAP) $\min \sum_{i=1}^{n} \sum_{j=1}^{n} \sum_{k=1}^{n} \sum_{l=1}^{n} c_{i j k l} x_{i j k l}$

subject to

$\sum_{i=1}^{n} x_{i j}=1, j=1, \ldots, n$,

$\sum_{j=1}^{n} x_{i j}=1, i=1, \ldots, n$

$x_{i j}=0,1, i=1, \ldots, n, j=1, \ldots, n$.

Eq. (1) states the objective function of the QAP problem and it minimizes sum of costs associated with facility assignment. Eq. (2) ensures that only any facility is assigned to only one place. Eq. (3) specifies that only each location is considered only for one place. Eq. (4) states that all variables are binay. The QAP problem is generally considered as an NP-Hard problem (Çela, 1998; Anstreicher, 2003) and cannot be solved using combinatorial optimization techniques. Therefore, an alternative solution is to use heuristic as well as meta-heuristics to tackle such problem although there are recently some studies concentrated on reformulation of QAP in an attempt to provide exact solution (Loiola et al., 2007).

\subsection{Tabu search}

During the past two decades, there have been various metaheuristics to solve QAP such as Tabu search, which was originally developed by Glover $(1986,1989,1990)$. Neighborhood searches take a potential solution to a problem and verify its immediate local opportunities, which is, solutions that are similar except for one or two minor details to detect an improved solution. Local search techniques tend to become stuck in suboptimal regions or on plateaus where several solutions are equally fit. Tabu search takes advantage of the performance of these methods by using memory structures, which explain the visited solutions or user-provided sets of rules. If a potential solution has been already visited within a certain short-term period or if it has already violated a rule, it is marked as "tabu" (forbidden) so that the algorithm would not reconsider that possibility, repeatedly (Hertz et al., 1995). Hussin and Stutzle (2011) presented a high performing stochastic local search algorithms 
for the QAP and their performance in dependence to the instance structure and size. Misevičius (2003) proposed a modified simulated annealing algorithm for the QAP - M-SA-QAP. They examined their algorithm on a number of instances from the library of the QAP instances - QAPLIB and reported that the proposed algorithm seemed to be superior to earlier versions of the simulated annealing for the QAP. Saifullah Hussin and Stützle (2014) compared the performance of Tabu search vs. simulated annealing as a function of the size of quadratic assignment problem instances. They reported that the assertion whether one algorithm is better than the other could depend strongly on QAP instance size even if one focuses on instances with otherwise same characteristics. Wilhelm and Ward (1987) applied simulated annealing to solve QAP problem. Fig. 1 shows the structure of Tabu search method.

Step 1. Let $S$ be the initial feasible solution and $Z$ its objective function value; then, set $S^{*}=S$, $\mathrm{Z}^{*}=\mathrm{Z}$, max short-term memory $(\mathrm{STM})=5$, and max iteration $=1,000$; iter $=1$. Best $\mathrm{O}$ value $=\mathrm{O}$ value.

Step 2. Random $(i, j)=$ rand/Long-term memory $(\operatorname{LTM})(i, j),(n 1, n 2)=$ the indices of maximum value in random.

Step 3. If there is none $(\mathrm{n} 1, \mathrm{n} 2)$ in STM matrix, change $\mathrm{n} 1$ and $\mathrm{n} 2$ locations; otherwise, repeat step 2.

Step 4. Insert $\mathrm{n} 1$ and $\mathrm{n} 2$ in STM and release the last indices from STM (e.g., m1, m2); and LTM(m1, $\mathrm{m} 2)=\operatorname{LTM}(\mathrm{m} 1, \mathrm{~m} 2)+1$.

Step 5. Calculate the objective function value $(Z)$ of the new permutation.

Step 6. If $Z \leq Z^{*}$, then $Z^{*}=Z, S^{*}=S$, and iter $=$ iter +1 .

Step 7. If iter $\leq \max$ iteration, then repeat step 2; otherwise, print $Z^{*}$ and $S^{*}$.

Fig. 1. Tabu search algorithm

\subsection{Simulated annealing}

Simulated annealing (SA) is a generic probabilistic metaheuristic for combinatorial optimization problem of locating a good approximation to the global optimum of a given function in a relatively large search space. The method is often implemented when the search space is discrete such as QAP problems. For certain problems, SA may be more efficient than exhaustive enumeration rather than the best possible solution. Paul (2010) reported that for a number of varied problem instances, SA could perform better for higher quality targets while TS performs better for lower quality targets. Fig. 2 shows details of the SA method.

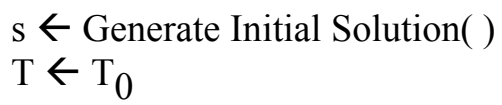

while termination conditions not met do

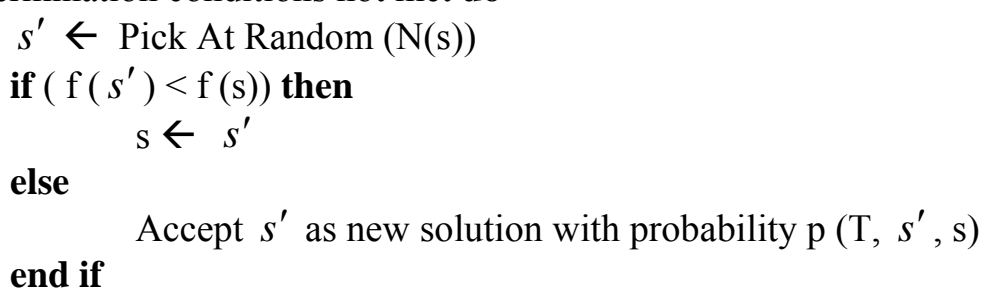

Fig. 2. The structure of SA method

The proposed study of this paper propose a hybrid of Tabu and SA method. Fig. 3 shows details of Pseudu code of the proposed study. 
step1 :

initialize $S$ as initial solution and $z=$ evaluate objective fuction

step1. $3: S^{*}=S$ and $Z^{*}=Z$;

$\mathrm{STM}=5 ; / /$ max short-term memory

maxiteration $=1000$; iter $=1$; best value $\mathrm{O}=\mathrm{O}$ value

step2: randomize

step2.1: for $\mathrm{i}=1$ to $\mathrm{n}$ do

for $\mathrm{j}=1$ to $n$ do

RANDOM $(i, j)=$ rand/LTM;

step2.2 : $(i, j)$ and $(n 1, n 2)=$ index of $($ RANDOM $\in S T M)$;

step3 : $\mathrm{T}=0$;

for $\mathrm{i}=1$ to $\operatorname{size}(\mathrm{STM}, 1)$ do

for $\mathrm{j}=1$ to $\operatorname{size}(\mathrm{STM}, 2) \mathrm{do}$;

$\operatorname{if}((n 1, n 2)==\operatorname{STM}(i, j))$

$\mathrm{T}=1$; repeat Step 2

if $(\mathrm{T}=0)$

\{

temp $=\mathrm{n} 1$;

$\mathrm{n} 1=\mathrm{n} 2$;

$\mathrm{n} 2=$ temp

\}

\section{Step 4:}

$\mathrm{m} 1=\operatorname{size}(\mathrm{STM}, 1)$;

$\mathrm{m} 2=\operatorname{size}(\mathrm{STM}, 2)$;

$(\mathrm{n} 1, \mathrm{n} 2)=\operatorname{STM}(\mathrm{m} 1, \mathrm{~m} 2)$;

$\operatorname{LTM}(\mathrm{m} 1, \mathrm{~m} 2)=\operatorname{LTM}(\mathrm{m} 1, \mathrm{~m} 2)+1$;

step5: $\mathrm{z}=$ evaluate objective function;

step 6 :

if $\left(\mathrm{z}<=\mathrm{z}^{*}\right) ; \mathrm{z}^{*}=\mathrm{z}$

\{

$\mathrm{S} *=\mathrm{S}$

iter $=$ iter +1

\}

step7: if (iter $<=$ max iteration) ; repeat step 2 ; else print $\mathrm{z}^{*}$ and $\mathrm{S}^{*}$

Fig. 3. Hybrid Tabu-Simulated Annealing Pseudo-code

\section{The results}

In this section, we present details of the implementation of the proposed study (TABUSA) and compares the results with pure Tabu search method (TS). Using some well known problems from QAPLIB generated by Burkard et al. (1997), two methods of TABUSA and TS are both coded on MATLAB and they are compared with relative percentage deviation (RPD) for all instances. Table 1 shows details of our results. 
Table 1

The summary of comparison of TABUSA versus TS

\begin{tabular}{ccccc}
\hline Name of instances & $\mathrm{n}$ & BKS & RPD of TS solutions & RPD of Hybrid Tabu-SA solutions \\
\hline Nug 12 & 12 & 578 & 0.00 & $\mathbf{0 . 0 0}$ \\
Nug 14 & 14 & 1014 & 0.39 & $\mathbf{0 . 0 0}$ \\
Nug 15 & 15 & 1150 & 0.87 & $\mathbf{0 . 0 0}$ \\
Nug 16a & 16 & 1610 & 1.37 & $\mathbf{0 . 0 0}$ \\
Nug 16b & 16 & 1240 & 0.00 & $\mathbf{0 . 0 0}$ \\
Nug 17 & 17 & 1732 & 0.69 & $\mathbf{0 . 0 0}$ \\
Nug 18 & 18 & 1930 & 1.04 & $\mathbf{0 . 0 0}$ \\
Nug 20 & 20 & 2570 & 1.56 & $\mathbf{0 . 3 8}$ \\
Nug 25 & 25 & 3744 & 1.55 & 0.12 \\
Bur26a & 26 & 5426670 & $\mathbf{0 . 0 9}$ & $\mathbf{0 . 0 0}$ \\
Bur26b & 26 & 3817852 & 0.19 & $\mathbf{0 . 0 2}$ \\
Bur26c & 26 & 5426795 & 0.26 & $\mathbf{0 . 0 1}$ \\
Bur26d & 26 & 3821225 & 0.02 & $\mathbf{0 . 0 1}$ \\
Bur26e & 26 & 5386879 & 0.03 & 0.00 \\
Bur26f & 26 & 3782044 & 0.05 & 0.03 \\
Bur26g & 26 & 10117172 & $\mathbf{0 . 0 1}$ & $\mathbf{0 . 0 0}$ \\
Bur26h & 26 & 7098658 & 0.01 & $\mathbf{2 . 7 1}$ \\
Tai25a & 25 & 1167256 & 4.26 & $\mathbf{3 . 8 2}$ \\
Tai30a & 30 & 1818146 & 4.75 & $\mathbf{4 . 1 2}$ \\
\hline Tai40a & 40 & 3139370 & 6.12 & $\mathbf{0 . 6 6}$ \\
\hline Tai50a & 50 & 4938796 & 6.49 & \\
\hline
\end{tabular}

As we can observe from the results of Table 1, the proposed study of this paper is capable of finding optimal solutions in most cases and performs better than TS method. Fig. 4 shows details of error for two methods.

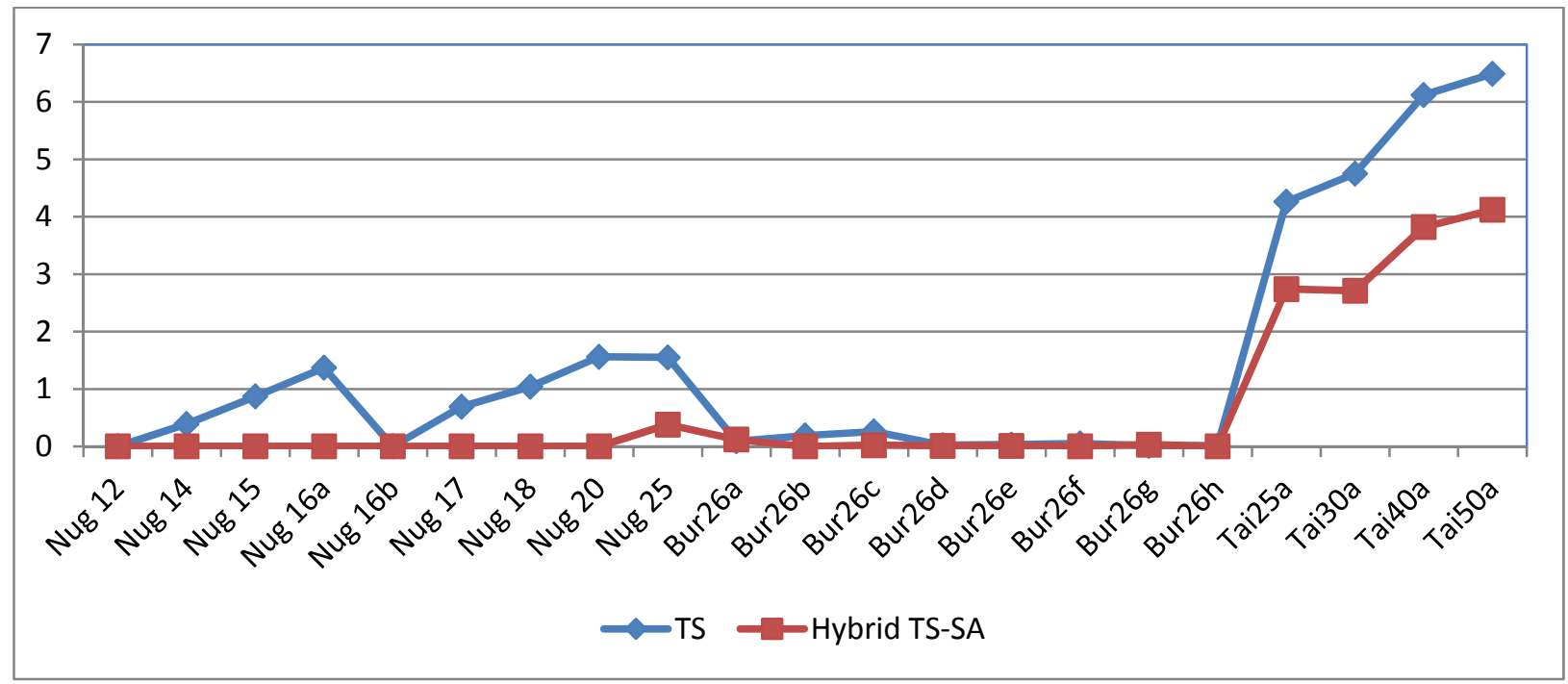

Fig. 4. Comparison of RPDs for TABUSA versus TS

\section{Conclusion}

In this paper, we have presented a hybrid method based on two well known methods, Tabu search and simulated annealing to solve QAP problem. The proposed model fo this paper has been implemented on some benchmarks and the results have confirmed that the new hybrid method could perform better than pure Tabu search method to solve QAP problems. However, we should cuatios about the findings since we may need to apply the proposed model of more instances to make more reliable conclusion. 


\section{Acknowledgement}

The authors would like to thank the annonymous referees for constructive comments on earlier version of this paper.

\section{References}

Ahmed, Z. H. (2013). A New Reformulation and an Exact Algorithm for the Quadratic Assignment Problem. Indian Journal of Science \& Technology, 6(4), 4368-4377.

Anstreicher, K.M. (2003). Recent advances in the solution of quadratic assignment problems. Mathematical Programming Series B, 97, 27-42.

Burkard, R. E., Karisch, S. E., \& Rendl, F. (1997). QAPLIB-a quadratic assignment problem library. Journal of Global Optimization, 10(4), 391-403.

Burkard, R. E. (2013). Quadratic assignment problems (pp. 2741-2814). Springer New York.

Çela, E. (1998). The Quadratic Assignment Problem: Theory and Algorithms. Kluwer Academic Publishers, Dordrecht.

Forghani, K., \& Mohammadi, M. (2012). Integrated quadratic assignment and continuous facility layout problem. International Journal of Industrial Engineering Computations, 3(5), 787-806.

Glover, F. (1986). Future paths for integer programming and links to artificial intelligence. Computers \& Operations Research, 13(5), 533-549.

Glover, F. (1989). Tabu Search - Part 1. ORSA Journal on Computing, 1(2), 190-206.

Glover, F. (1990). Tabu Search - Part 2. ORSA Journal on Computing, 2(1), 4-32.

Hertz, A., Taillard, E., \& De Werra, D. (1995). A tutorial on tabu search. InProc. of Giornate di Lavoro AIRO, 95, 13-24.

Hussin, M. S., \& Stutzle, T. (2011, December). High performing stochastic local search algorithms for the QAP and their performance in dependence to the instance structure and size. In Hybrid Intelligent Systems (HIS), 2011 11th International Conference on (pp. 139-144). IEEE.

Koopmans, T. C., \& Beckmann, M. J. (1957). Assignment problems and the location of economic activities. Econometrica, 25, 53-76.

Loiola, E. M., De Abreu, N. M. M., Boaventura-Netto, P. O., Hahn, P., \& Querido, T. (2007). A survey for the quadratic assignment problem. European Journal of Operational Research, 176(2), 657-690.

Misevičius, A. (2003). A modified simulated annealing algorithm for the quadratic assignment problem. Informatica, 14(4), 497-514.

Paul, G. (2010). Comparative performance of tabu search and simulated annealing heuristics for the quadratic assignment problem. Operations Research Letters, 38(6), 577-581.

Saifullah Hussin, M., \& Stützle, T. (2014). Tabu search vs. simulated annealing as a function of the size of quadratic assignment problem instances. Computers \& Operations Research, 43, 286-291.

Tasgetiren, M. F., Pan, Q. K., Suganthan, P. N., \& Dizbay, I. E. (2013, April). Metaheuristic algorithms for the quadratic assignment problem. InComputational Intelligence In Production And Logistics Systems (CIPLS), 2013 IEEE Workshop on (pp. 131-137). IEEE.

Tseng, L. Y., \& Liang, S. C. (2006). A hybrid metaheuristic for the quadratic assignment problem. Computational Optimization and Applications, 34(1), 85-113.

Wang, J. C. (2007, November). Solving quadratic assignment problems by a tabu based simulated annealing algorithm. In Intelligent and Advanced Systems, 2007. ICIAS 2007. International Conference on (pp. 75-80). IEEE.

Wilhelm, M. R., \& Ward, T. L. (1987). Solving quadratic assignment problems by 'simulated annealing'. IIE transactions, 19(1), 107-119. 